\title{
Future perspectives of digital pathology
}

\author{
Monika Prochorec-Sobieszek
}

Technological advances have enabled innovative solutions to be achieved in pathology based on digital imaging, now superseding those of conventional microscopy. Digital pathology has been defined as 'virtual microscopy' and depends on computer-generated digital imaging of microscope slides (WSI — whole slide imaging) which are in turn created, reviewed, managed, shared, analysed and interpreted. Such WSI systems and digital consulting platforms are now used for teaching, scientific research, telepathology / teleconsultation and diagnostics. They also permit easy and interactive sharing of WSI that can be integrated into other medical information systems. The software for automated image analysis and computer aided diagnosis can thereby make highly accurate diagnoses and help standardise study findings. Despite the technique's many advantages, its noted drawbacks include high equipment and software costs, image quality issues of standardisation and most importantly, that pathologists are reluctant to use it routinely for making diagnoses.

NOWOTWORY J Oncol 2016; 66, 4: 277-285

Key words: digital pathomorphology, whole slide images, telepathology/teleconsultation, education, research, archiving, digital diagnostics, IT technologies

\section{Introduction}

The quality of healthcare delivered to cancer patients begins at the cellular level and is closely linked with making the correct pathomorphological diagnosis, taking into account prognostic and predictive factors. Regardless of the pathologist's key role when diagnosing cancer, pathomorphology has not really yet taken advantage of the progress accomplished in information technology (IT); in contrast with its being adopted for radiology, urology and cardiology. Digital pathology comes down to using digital images of whole microscope slides (WSI) for various purposes. WSI is often termed 'virtual microscopy' and is the computer equivalent of conventional microscopy. Practically, it consists of two processes: the first being to create digital images by scanning microscope slides, whilst the second is through using dedicated software (browsers) for viewing and analysing such digital images [1, 2].

Digital pathology can be broadly defined as creating, viewing, managing, sharing, analysing and interpreting digital images of microscope slides. Amongst its other applica- tions, this includes teaching, scientific research, archiving, telepathology / teleconsultation and routine diagnostics. Such a digital transformation in pathology was made possible due to the continuous modernisation of scanning devices, the increasing computers power and the transfer rate coupled with introducing new software solutions and technologies for collecting large amounts of data. One of the important advantages of digital pathology is providing the means to better integrate diagnosis and treatment of cancer by facilitating interaction between multidisciplinary teams of doctors involved in diagnosing and treating these diseases [1-11].

Pathomorphology is based on interpreting images of cells and tissues at higher resolutions then the resolution of the human eye. Over many years light microscopy was the only instrument capable of doing this, where improvements had been made in optical devices and in enhancing the resolution of microscope images [12]. Indeed, routine pathological diagnosis is still performed on slides by light microscopy as standard. 
The optical techniques used in pathology nevertheless began to gradually change during recent years with the advent of installing digital cameras for taking photographs in light microscopes, along with video cameras for assessing microscope slides in real time. It became thereby possible to introduce teleconsultation of microscopic images, via a computer network, between pathologists working in remote locations and to perform automated imaging intraoperatively; however, the images thus generated being of a relatively low resolution [13-16]. Within about the last ten years, advances in technology have enabled scanners to be used which have allowed digital images to be made of whole microscope slides i.e. WSI. This combines the advantages of camera images obtained in realtime mode (availability of WSI) with those of high resolution digital cameras $[12,16]$.

Despite the many advantages of virtual microscopy, its limitations should also be recognised; being especially paramount whenever it is to be introduced for routine pathological diagnosis. This article discusses the pros and cons of this technology and its application to clinical and non-clinical practice.

\section{Technologies}

The technological basis underpinning digital pathology is the WSI system, consisting of scanners and the IT infrastructure device. The scanner includes: an optical microscope, one or more digital cameras for taking pictures, ordinary and fluorescent light sources, slide loader, a workstation and software that permits the user to adjust the image to the specific desired depth comparable to conventional light microscopy. The IT infrastructure includes: computer and software for image acquisition, processing and file management, a server with external memory for digital image database and a secure and reliable computer network for linking workstations, servers and external memory storage of the scanned images database [4].

\section{Microscope slide scanners}

There are many scanners commercially available from different manufacturers whose technical specifications vary. One of these is the capacity of simultaneous loaded microscope slides. Different scanning techniques are also used for creating images: line scanning (based on precisely moving a motorized stage with the object line by line) or area scanning with a CCD camera (charge coupled device) which successively takes digital pictures as a series of rectangular tiles of the object field. The strips or tiles are assembled into a WSI either concurrently or after the scanning is finished [12]. Different scanner magnifications are obtained from various lenses and numerical apertures; most commonly used are a 20 and 40 magnification. Some scanners have the facility for performing multi-layer scans using z-stacking technol- ogy. Although making 3D images substantially extends scanning times, the advantages are the ability to analyse cytology smears, frozen sections and other thickened objects requiring an assessment on different layers. Grading mitosis is likewise expedited by this technique. Frequently, scanners have a fluorescent light source as well as various sensors for improving image quality thus enabling cells and tissues to be scanned whose individual structures are stained fluorescently and then converted into high-resolution colour images. Scanners also vary according to the file formats of the scanned images that often include either blocked proprietary formats (owned by the manufacturer) or open formats such as a standard jpeg 2000, or standard DICOM (Digital Imaging and Communications in Medicine) compression to jpeg (2000) [17] .

Because WSI requires both high resolution and precision for transforming scanned objects into digital images, the file storage space needs to be very large, ranging from tens of MG (megabytes) to tens of GB (gigabytes). Furthermore, such file sizes need to accommodate the actual surface size of the tissue slide. A typical virtual slide that is 1,600 megapixels would require about $4.6 \mathrm{~GB}$ of memory, where these WSI sizes are much larger than those used for radiology [18]. Various techniques are currently employed for reducing the size of such huge files, for instance algorithms devised for reducing scan areas to the dimensions of the detected tissue, and the image compression method [4]. The scan time for slides depends on the tissue sample size and the actual stages of the scanning for capturing all the required data from the slide and then constructing an appropriate image. For example, a single layer scan of $15 \times 15 \mathrm{~mm}$ sample at $40 x$ magnification takes from several to tens of minutes depending on the scanner type [19].

The quality of digital imaging, upon which a correct interpretation can be made, is based on various factors [20] as follows:

- Appropriate fixation and handling of tissue material without the technical problems associated with making tissue sections, staining and in sealing the slide.

- Optical scanner set up, focusing methods, colour management, white balance and contrast.

- The precision in the merging of image components and the extent of compression required after digital imaging.

- The completeness of the final digital image, which should encompass all the tissue fragments found within the microscopic slide.

- The quality of the computer's display or projector used for presenting a digital image mediated by aspects like screen resolution, colour accuracy, brightness and contrast.

- Servicing and presentation of digital images depending on the used browser (scroll fluency, a facility for varying the focus), or IT infrastructure (short access times). 
Digital imaging has the following advantages over the conventional microscopy technique [3]:

- Their quality does not change over time.

- They allow simultaneous viewing by many pathologists around the world via the Internet.

- They can be shared for digital image analysis and morphometric measurements.

- They are more mobile than microscope slides and can be easily extracted from digital archives for various clinical and non-clinical uses.

- They can be easily integrated into other electronic patient records.

- The Digital Image Viewer allows simultaneous viewing of several images, thereby simplifying comparisons between any selected structures under different staining.

- The ability for simultaneous observation of images on the monitor and preview screen, thereby permitting optimal orientation and easy navigation towards any interesting locations on any given slide.

\section{IT infrastructure}

After being scanned, the digital images of microscope slides are provided to the user and then send to the Laboratory Information System (LIS); integration with the latter being vital. Identification and archiving of images along with their integration into LIS enables bar-coding to be used. Storing WSI files requires storage of a suitable capacity. Also, the memory size depends on the application intended for the digital images. Storing a limited numbers of images for the purposes of consultation, research or teaching does not however require too much memory, whilst large scale scanning does; for example the workload of a medium sized laboratory requires a huge amount of space with an external memory of up to 50 TB (terabytes) per year, with no space reserved for backups [19]. Depending on the numbers of scanners and users then controllers and fibre connections may be required. Fast data access can be enabled by using SSD disks (solid-state drive). Because not all digital images are required to be immediately available, the older images can be archived to slower, but cheaper storage media such as magnetic tapes. The quality of the systems' display, however impacts on the assessment of digital images; the most important parameter being the resolution which determines both image quality and field size. Monitors with a resolution of $1600 \times 1200$ pixels, can display only about $20 \%$ of the total image possible when viewed by microscope [21]. The digital images quality is also affected by colour calibration, contrast and brightness.

Network bandwidth is also a limitation when images are transmitted, in that it needs to be sufficiently big to quickly transfer large files containing digital images. Usually, a network bandwidth of $100 \mathrm{Mb}$ (megabytes per second) is enough. Most browsers uses efficient mechanisms for ensuring a suitable speed for image viewing. Instead of downloading entire image files, only sections of interest are selected from the whole image in disk storage. This information is then stored in the cache allowing it be rapidly accessed Many browsers initially display the first image at a lower resolution before downloading all the details required for presenting a final image in the high resolution.

In circumstances where the images need to be simultaneously available to multiple users, (for instances of digital consultation sessions or educational courses), then specially configured servers are used which have the facility for sharing images from the cache memory. Today, most manufacturers save scanned images to files using their own formats. Customised formats are an inconvenience to end users, as this requires installing dedicated browsers, which is a major obstacle in the digital microscopy market. It is therefore proposed that formats should be standardised to JPEG 2000 [22].

\section{The use of digital imaging in pathomorphology}

The ever advancing resolution of digital images coupled with higher scan speeds, the more convenient-to-use browsers and the ability to transfer images to different locations (telepathology) have all ensured that WSI systems have found many applications in pathology [4]. Notwithstanding its many advantages, digital images are currently used mainly for applications indirectly related to diagnostics such as teaching, archiving, multi-disciplinary meetings and research. Within diagnostics itself, only teleconsultation and automated intraoperative examination have become adopted. At present, routine pathomorphological digital diagnostics is neither so widely accepted nor widespread as compared to digital diagnostic radiology [23].

\section{Digital diagnostics}

The current state for this technology already allows routine digital diagnostics to be performed, yet only a few laboratories in the world are entirely digital. There are various reasons for this. Laboratory work involved in using this technology line to prepare WSIs takes more time than the traditional organisation of workloads, because of the additional procedure required for creating digital images. Despite the many advantages of digital diagnostics, most pathologists feel more confident when using light microscopes for making diagnoses, especially if they had previously received their education in conventional methods [23]. Furthermore, in spite of all the facilities available for automated image analysis, making diagnoses is more rapid and simple when using light microscopes than using computers. A study by Randell et al. [24] has demonstrated that routine diagnostics performed by a pathologist using digital tools is on average $60 \%$ slower than when a microscopic diagnosis is made. Upon introducing routine digital 
diagnostics, a large investment in IT infrastructure is required including setting up the hardware and software as well as additional staff training [19]. It should also be noted that even the most modern scanners still do not fully cover all the functions of modern optical microscopes [23].

Studies published from medical centres performing pathomorphology have demonstrated a high correlation between routinely used digital diagnostics and conventional microscopy, where the percent error is of the order $1-5 \%$ [24-32]. These discrepancies arise mainly from inferior image quality and the pathologist being inexperienced in using digital techniques. A Dutch laboratory in Heerlen found an $82.1 \%$ agreement when scanning $20 \%$ of those surgical biopsies being undertaken there [33]. The time needed for evaluating digital images appears to be a recurrent theme in many reported studies. Smaller biopsies occupy less space on a slide and thus they can usually be scanned quickly and then viewed. However, digitisation of larger tissue sections not only takes more time, but the sample preparation may encroach beyond the slide coverslip, which makes obtaining the correct focus required for scanning more difficult. It should also be stressed that poorly stained sections of small tissue pieces are not always 'recognised' by the scanning systems, although studies indicate that WSI systems can be effectively used for core needle biopsies [34].

WSI can also be used for cytopathology, although currently it is employed more for teaching purposes than diagnostic ones. The reason for this being that the thickness of the cytological smears on a slide requires that scans are taken for many layers [35]. In order to obtain an adequate quality of digital images, it is necessary to conduct the longduration z-stacking technique [36].

An important facility provided by digital pathology is for improving the accuracy of diagnosis through digital image analysis. Software intended for quantifying protein expression by immunohistochemical means, based on digital imaging, has lead to an improved assessment of immunoreactivity [37]. In immunohistochemistry, imaging analysis is most commonly performed for an integrated assessment of receptor expression that includes those for oestrogen, progesterone, protein HER2 / Neu (human epidermal growth factor receptor 2) and the proliferative Ki67 index in breast cancer [38]. Analyses based on WSI images shows a higher degree of agreement when measuring expression of HER2 / Neu with the results of FISH gene amplification (fluorescence in situ hybridization) in breast cancer compared with the traditional assessment by microscopy [39]. Nevertheless, digital analysis is not fully effective for all its applications; for instance when measuring mitotic index, using the most advanced image analysis software, only a $80-85 \%$ agreement is reached when compared to conventional microscopy [40]. This aforementioned digital analysis is a very useful IT tool, but immunohistochemistry measurements represents only a small proportion of the pathologist's diagnostic workload. There is much recent interest in IT applications for making computer aided diagnosis (CAD), that are based on WSI systems [41-43]. Combining WSI with the tools for automated image analysis enables an objective assessment to be made of factors governing the eligibility of patients towards an appropriate therapy, such as histological tumour grading [44] as well as an automated selection of those areas requiring investigation i.e. the so-called 'Hot Spots'; a term describing the most active areas of cancer proliferation [45]. Making a pathological diagnosis is further enhanced by the ability to search and compare results with images from digital databases, however this way so far has not been adopted for routine diagnostics [46].

\section{Telepathology}

Digital diagnostics that uses telepathology, i.e. transfer of digital images from remote locations is performed when consultation is required for difficult to diagnose cases (termed 'second opinions') as well as for remote intraoperative examination. A rapid diagnosis of frozen specimens by digital means constitutes a support for those hospitals where intraoperative testing is unavailable in circumstances where there are no pathologists present. Telepathology also facilitates teleconsultation. An advantage of this technology is to reduce sample testing time; they can be performed within a few hours as compared to the traditional way of delivery by post, which usually takes several days. This technology depends on specialised software, the so-called 'digital platform' where a pathologist can send a digital image via the Internet to another consultant pathologist. It is also possible to discuss any given case on the Internet, where one consulting party and can suitably move the image and change the magnification, whilst other parties can view images on their computer screens, tablets or smartphones in real time. Through these means, meetings can be held by experts from different parts of the world specialising in particular areas of pathology in order to consult with each other on difficult cases. The WSI allows pathologists to work remotely, where tasks can for example be performed, at home or anywhere else in the world $[47,48]$.

\section{Multidisciplinary meetings}

An important application of the WSI systems, especially for cancer patients, are multidisciplinary meetings. Pathologists are able to discuss cases with doctors from other specialisations (eg. oncologists, surgeons, radiologists) from almost anywhere by means of computer generated digital images of microscope slides which can be magnified, moved and annotated, as and when required [3].

\section{Quality control}

Another use of telepathology and WSI imaging are in allowing external quality control of staining and in making 
histopathological diagnoses. For these to be assured, regular reviews of selected cases are conducted by those hospital units responsible for accreditation. Sending microscope slides by post is time consuming and there is the attendant risk to be considered of loss or damage to such specimens. By performing these tasks via digital means, the process can be speeded up without the need for sending any specimens. At present some digital WSI platforms include software designed for internal quality control of digital images generated from microscope slides [48].

\section{Validation}

Using the WSI system for routine diagnosis requires validation. It is necessary that equivalence in diagnosis be demonstrated in specimens between those evaluated by digital imaging and those based on conventional light microscopy. The agreement between these methods depends upon the performance of the computer technology and the experience of pathologists in making diagnosis by digital means. It should be noted that when making diagnoses by conventional microscopy, differences in the interpretation of individual cases can occur, which will increase to more problematic any specimen is found to be. Recommendations exist for using WSI systems in diagnostics including, amongst others, guidelines from the College of American Pathologists (CAP), the Digital Pathology Association and the Royal College of Pathologists [49]. According to CAP guidelines, internal quality control should be undertaken for any laboratory deciding to carry out digital diagnostics. The WSI system should be validated in its entirety by specialists and pathologists in such fashion as to mimic the process of routine pathological diagnosis in a given medical establishment. CAP recommends validating at least 60 cases of one specimen type eg. fixed tissue slides stained with hematoxylineosin, cytological specimens or frozen sections from intraoperative procedures; where selected cases must reflect the scope of tests performed in routine pathology practice. During the validation, a time interval of at least two weeks should be kept between the analyses of microscope slides and the digital images.

\section{Education}

Pathology was traditionally taught by the shared viewing of slides under two or multi-headed microscopes which limited the number of students and pathologists that could simultaneously view a given specimen. In addition to viewing slides under the microscope, it was also possible to use software programmes containing static digital images of microscope slides. Education indeed became one of the first applications for WSI technologies and platforms because of the ease of creating and managing the repositories of digital images. WSI systems deliver the same digital images as microscope slides which can thus be used by lecturers for teaching an unlimited number of students, even at remote locations. In this way, digital platforms fulfil the operation of multi-headed microscopes whilst at the same time they permit, through using professional software images, more interactive facilities like annotation, asking of questions, using various links, video clips and setting on-line tests/ exams for passing certified qualifications in areas of underand post-graduate education.

Teaching by digital means is more standardised because the educational materials, including sets of digital images of microscope specimens, are the same for all students and pathologists. Repositories of digital images are of particular importance in teaching about rare disease cases, having small biopsies, cytology specimens and any unique consultation cases where it is difficult to prepare microscopic slides for large numbers of students. Investing in WSI-based systems for education is cost-effective because universities do not have to buy hundreds of microscopes and prepare collections of assorted slides, which can easily become damaged. In addition, students are no longer tied to the laboratory at their medical schools; they can use repositories from far-away locations at any place and time through using computers, tablets or smart phones possessing an Internet connection by logging on to digital platforms and thus gaining suitable access to educational materials.

Nonetheless, digital based teaching also has some limitations. Students and pathologists base their knowledge on digital images without using microscopes, which could affect the quality of any future diagnoses if made by light microscopy. Digital education is totally dependent on a well-functioning IT infrastructure and any failures could slow down the system and thereby adversely affect learning. In addition, the image resolution of WSI with a good scanner may be inferior to images seen under a good microscope, even though they appear good enough for teaching purposes. Users of WSI also tend to be dissatisfied with the speed of the browser and the delays in downloading huge files with images from a slow-working network.

Teaching laboratories that use virtual microscopy exist at many universities around the world. It seems that students have quickly accepted being taught by the WSI system in preference to conventional teaching methods that use microscopes. The test and examination grades achieved after virtual training have been similar [50-52].

\section{Digital archiving}

Archiving in pathology involves, amongst other things, storage of microscope slides. This method of archiving causes many problems such as the need for large storage facilities with reinforced floors, the vulnerability of fragile microscope slides to damage, stain-fading over time and logistical issues concerning slide rental and return. Compared 


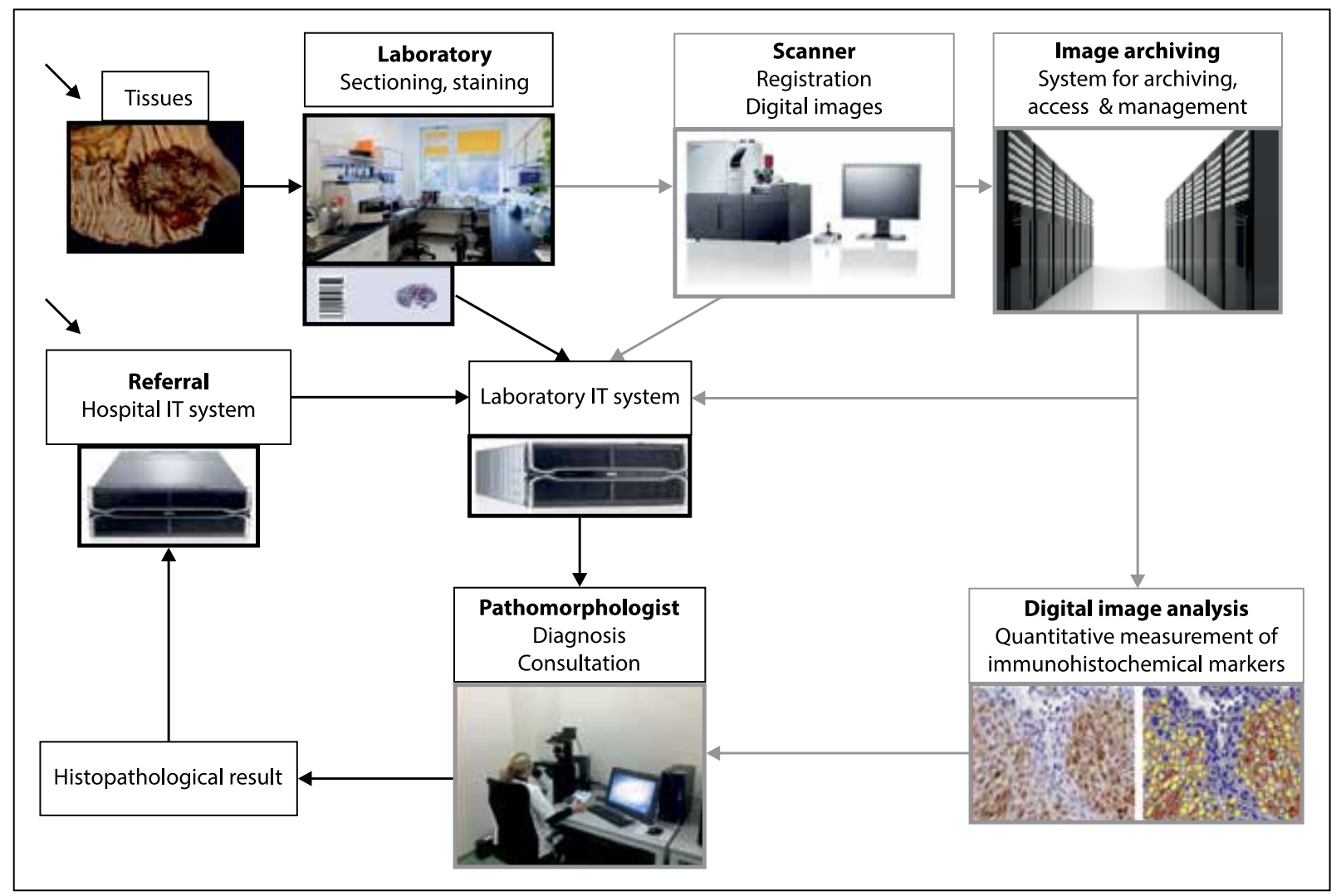

Figure 1. Management of Digital Pathomorphology systems

to these traditional methods of archiving, digital archiving based on WSI images has many advantages as follows:

- WSI Images are stored without deteriorating quality (especially immunofluorescent specimens).

- It is easy to download images from WSI archives for review, teaching purposes, research, clinical-pathological meetings and external quality control.

- WSI images can be included in reporting pathological and medical case histories, within hospital information systems.

- Digital archiving may contain specimens that are consultative in nature before they are relayed back to their place of origin. These digital images are useful for when any comparisons are required in subsequent patient testing. They can also prove to be interesting material for teaching purposes.

- The digital images are frequently the only patient documentation left over after cellular material from slides has been'scraped off' in cases where ensuing molecular testing becomes necessary [12].

\section{Research studies}

The WSI platform enables the easy and rapid replacement of thousands of digital images, along with any annotations made between researchers working on national and international projects. The largest use is for image analysis software that takes morphometric measurements and quantifies the expression of proteins identified by immunohistochemistry and gene amplification. Of vital importance is analysing digital images of tissue microarrays (TMA). Contained within a single microscope slide and then on one digital image, samples from many patients can be analyzed as a whole and in standardised fashion, and likewise each sample can constitute excellent material for individual viewing and analysis. The digital image archives form the basis for doing research and act as data warehouses containing vast amounts of information. They are even more valuable when integrated into a database of histopathological diagnoses and also with a repository of fresh or fixed tissue, thereby creating the structure for biobanks $[53,54]$.

\section{Clinical trials}

WSI technology is also increasingly important for clinical trials. In order to assure correct pathological diagnoses and to standardise a given target population according to set criteria therein for microscope slides and paraffin block samples, cancer patients have to be re-evaluated in designated laboratories by experienced pathologists. Currently, microscope specimens are posted to various centres, which is time consuming and prolongs patient classification. By means of 
WSI platforms and the transfer of digital images together with teleconsultation, it is possible to shorten, simplify and standardise the process of qualification. When considering clinical trials involving large groups of patients, there is also the possibility of standardising quantitative measurement of immunohistochemically-labeled proteins intended as therapeutic targets through applying automated analysis of digital images [55]. This thereby creates huge digital repositories of standardised diagnoses and specimens that can be used for future research on new therapeutic targets.

\section{Conclusions}

WSI technology as it now stands basically represents the future trend in pathomorphology. WSI systems have found many uses in modern teaching, diagnosis and research work. The WSI platform has enabled easy and interactive sharing of digital images and afforded a superior integration of digital images with other information systems. Improvements in image analysis software and computer aided diagnosis has now become accepted in routine diagnostic procedures in those situations requiring a more accurate and reproducible grading of some tumours as well as quantitative measurement of prognostic and predictive biomarkers. In order to achieve diagnostic equivalence when using digital imaging, it is necessary that the quality of preparing microscope slides is standardised (i.e. fixing, sectioning, staining and sealing) and matched by standardising the taking of digital images (i.e. resolution, number of layers scanned, distances between scanned layers and colour calibration). An important consideration for end users is for having open, unified format of the files into which the digital images are stored in.

It seems that the digitisation of pathology will not be as quickly accepted as the transition already achieved in digital radiology. The drawbacks are the large investments required in hardware and software, the slowing down of the diagnostic process, difficulties in scanning some samples and above all else, that pathologist are lack confidence of using WSI systems for routine diagnostics. Introducing digital pathology should really start for preclinical applications such as teaching, research, archiving and teleconsultation. For digital pathology to become part of routine diagnosis, it still appears that improved technology is needed coupled with changing the attitudes of pathologists.

\section{Aknowledgements}

Sincere thanks to Jerzy Mrozek MSc for providing technical and IT support throughout.

Conflicts of interest: The author declares no conflicts of interest
Prof. Monika Prochorec-Sobieszek, MD, PhD

Department of Pathology and Laboratory Diagnostics

Maria Sklodowska-Curie Memorial Cancer Center and Institute

of Oncology

ul. Roentgena 5, Warsaw, Poland

e-mail:monika.prochorec@interia.pl

Received \& Accepted: 6 April 2016

\section{References}

1. Pantanowitz L, Valenstein PN, Evans AJ et al. Review of the current state of whole slide imaging in pathology. J Pathol Inform 2011; 2: 36.

2. Leong FJ, Leong AS. Digital photography in anatomical pathology. J Postgrad Med 2004; 50: 62-69.

3. Al-Janabi S, Huisman A, Van Diest P. Digital pathology: current status and future perspectives. Histopathology 2012, 61:1-9.

4. Farahani N, Parwani AV, Pantanowitz L. Whole slide imaging in pathology: advantages, limitation, and emerging perspectives. Pathol Lab Med Int 2015, 7: 23-33.

5. Wiley CA, Murdoch G, Parwani A et al. Interinstitutional and interstate teleneuropathology. J Pathol Inform 2011; 2: 21.

6. Evans AJ, Chetty R, Clarke BA et al. Primary frozen section diagnosis by robotic microscopy and virtual slide telepathology: the University Health Network experience. Hum Pathol 2009; 40: 1070-1081.

7. Gifford AJ, Colebatch AJ, Litkouhi S et al. Remote frozen section examination of breast sentinel lymph nodes by telepathology. ANZ J Surg 2012; 82: 803-808.

8. Yagi Y, Gilbertson JR. Digital imaging in pathology: the case for standardization. J Telemed Telecare 2005; 11: 109-116.

9. Wilbur DC. Digital cytology: current state of the art and prospects for the future. Acta Cytol 2011; 55: 227-238.

10. Park S, Pantanowitz L, Parwani AV. Digital imaging in pathology. Clin Lab Med 2012; 32: 557-584.

11. Chlipala E, Elin J, Eichhorn $\mathrm{O}$ et al. Archival and retrieval in digital pathology systems. Digital Pathology Association 2011: 1-10. https:// //digitalpathologyassociation.org/.

12. Huisman A, Looijen A, van den Brink SM et al. Creation of a fully digital pathology slide archive by high-volume tissue slide scanning. Hum Pathol 2010; 41;751-757.

13. Weinstein RS. Innovations in medical imaging and virtual microscopy. Hum Pathol 2005; 36; 317-319.

14. Teodorovic I, Isabelle M, Carbone A et al. TuBaFrost 6: virtual microscopy in virtual tumour banking. Eur J Cancer 2006; 42; 3110-3116.

15. Weinstein RS. Prospects for telepathology. Hum Pathol 1986; 17; 433-434.

16. Baak JP, van Diest PJ, Meijer GA. Experience with a dynamic inexpensive video-conferencing system for frozen section telepathology. Anal Cell Pathol 2000; $21 ; 169-175$.

17. Rojo MG, Garcia GB, Mateos CP et al. Critical comparison of 31 commercially available digital slide systems in pathology. Int J Surg Pathol 2006; 14; 285-305.

18. Hipp JD, Fernandez A, Compton CC, et al. Why a pathology image should not be considered as a radiology image. J Pathol Inform 2011; 2: 26.

19. Stathonikos N, Veta M, Huisman A et al. Going fully digital: Perspective of a Dutch academic pathology lab. J Pathol Inform 2013; 4: 15.

20. Glatz-Krieger K, Spornitz U, Spatz A et al. Factors to keep in mind when introducing virtual microscopy. Virchows Arch 2006; 448: 248-255.

21. Treanor D, Jordan-Owers $\mathrm{N}$, Hodrien J et al. Virtual reality Powerwall versus conventional microscope for viewing pathology slides: an experimental comparison. Histopathology 2009; 55: 294-300.

22. Tuominen VJ, Isola J. The application of JPEG 2000 in virtual microscopy. J Digit Imaging 2009; 22: 250-258.

23. Feldman M.D. Whole slide imaging in pathology: what is holding us back? Patho Lab Med Int 2015; 7: 35-38.

24. Randell R, Ruddle RA, Mello-Thoms $C$ et al. Virtual reality microscope versus conventional microscope regarding time to diagnosis: an experimental study. Histopathology 2013; 62: 351-358

25. Thorstenson S, Molin J, Lundström C. Implementation of large-scale routine diagnostics using whole slide imaging in Sweden: digital pathology experiences 2006-2013. J Pathol Inform 2014; 5: 14. 
26. Pantanowitz L, Wiley CA, Demetris A et al. Experience with multimodality telepathology at the University of Pittsburgh Medical Center. J Pathol Inform 2012; 3: 45.

27. Al Habeeb A, Evans A, Ghazarian D.Virtual microscopy using whole-slide imaging as an enabler for teledermatopathology: a paired consultant validation study. J Pathol Inform 2012; 3: 2.

28. Al-Janabi S, Huisman A, Vink A et al. Whole slide images for primary diagnostics in dermatopathology: a feasibility study. J Clin Pathol 2012; 65: 152-158.

29. Nielsen PS, Lindebjerg J, Rasmussen J et al. Virtual microscopy: an evaluation of its validity and diagnostic performance in routine histologic diagnosis of skin tumors. Hum Pathol 2010; 41: 1770-1776.

30. Leinweber $\mathrm{B}$, Massone $\mathrm{C}$, Kodama $\mathrm{K}$ et al. Teledermatopathology: a controlled study about diagnostic validity and technical requirements for digital transmission. Am J Dermatopathol 2006; 28: 413-416.

31. Massone C, Soyer HP, Lozzi GP et al. Feasibility and diagnostic agreement in teledermatopathology using a virtual slide system. Hum Pathol 2007; 38: 546-554.

32. Koch LH, Lampros JN, Delong LK et al. Randomized comparison of virtual microscopy and traditional glass microscopy in diagnostic accuracy among dermatology and pathology residents. Hum Pathol 2009; 40: 662-667.

33. Al-Janabi S, Huisman A, Nap M et al. Whole slide images as a platform for initial diagnostics in histopathology in a medium-sized routine laboratory. J Clin Pathol 2012; 65: 1107-1111.

34. Campbell WS, Hinrichs SH, Lele SM et al. Whole slide imaging diagnostic concordance with light microscopy for breast needle biopsies. Hum Pathol 2014; 45: 1713-1721.

35. House JC, Henderson-Jackson EB, Johnson JO et al. Diagnostic digital cytopathology: are we ready yet? J Pathol Inform 2013; 4: 28.

36. Kaplan KJ.Telecytopathology for immediate evaluation of fine-needle aspiration specimens. Cancer Cytopathol 2010; 118: 115-118.

37. Fine JL, Grzybicki DM, Silowash R et al. Evaluation of whole slide image immunohistochemistry interpretation in challenging prostate needle biopsies. Hum Pathol 2008; 39: 564-572.

38. Lloyd MC, Allam-Nandyala P, Purohit CN et al. Using image analysis as a tool for assessment of prognostic and predictive biomarkers for breast cancer: how reliable is it? J Pathol Inform 2010; 1: 29.

39. Minot DM, Kipp BR, Root RM et al. Automated cellular imaging ystem III for assessing HER2 status in breast cancer specimens: development of a standardized scoring method that correlates with FISH. Am J Clin Pathol 2009; 132: 133-138.

40. Veta M, van Diest PJ, Willems SM et al. Assessment of algorithms for mitosis detection in breast cancer histopathology images. Med Image Anal 2015; 20: 237-248.
41. DiFranco MD, O'Hurley G, Kay EW et al. Ensemble based system for whole-slide prostate cancer probability mapping using color texture features. Comput Med Imaging Graph 2011; 35: 629-645.

42. Samsi S, Krishnamurthy AK, Gurcan MN. An efficient computational framework for the analysis of whole slide images: application to follicular lymphoma immunohistochemistry. J Comput Sci 2012; 3: 269-279.

43. Sertel O, Kong J, Shimada $\mathrm{H}$ et al. Computer-aided prognosis of neuroblastoma on whole-slide images: classification of stromal development. Pattern Recognit 2009; 42: 1093-1103.

44. Yeh FC, Parwani AV, Pantanowitz $L$ et al. Automated grading of renal cell carcinoma using whole slide imaging. J Pathol Inform 2014; 5: 23.

45. Lu H, Papathomas TG, van Zessen D et al. Automated Selection of Hotspots (ASH): enhanced automated segmentation and adaptive step finding for Ki67 hotspot detection in adrenal cortical cancer. Diagn Pathol 2014; 9: 216.

46. Akakin HC, Gurcan MN. Content-based microscopic image retrieval system for multi-image queries. IEEE Trans Inf Technol Biomed 2012; 16: 758-769

47. Ho J, Parwani AV, Jukic DM et al. Use of whole slide imaging in surgical pathology quality assurance: design and pilot validation studies. Hum Pathol 2006; 37; 322-331.

48. Pantanowitz L, Dickinson K, Evans AJ et al. American Telemedicine Association clinical guidelines for telepathology. J Pathol Inform 2014; 5: 39.

49. Pantanowitz $\mathrm{L}$, Sinard JH, Henricks WH et al. Validating whole slide imaging for diagnostic purposes in pathology: guideline from the College of American Pathologists Pathology and Laboratory Quality Center. Arch Pathol Lab Med 2013; 137: 1710-1722.

50. Dee FR, Meyerholz DK. Teaching medical pathology in the twenty-first century: virtual microscopy applications. J Vet Med Educ 2007; 34: 431-436.

51. Heidger PM Jr, Dee F, Consoer D et al. Integrated approach to teaching and testing in histology with real and virtual imaging. Anat Rec 2002; 269: 107-112.

52. Kumar RK, Velan GM, Korell SO et al. Virtual microscopy for learning and assessment in pathology. J Pathol 2004; 204: 613-618.

53. Hipp JD, Lucas DR, Emmert-Buck MR et al. Digital slide repositories for publications: lessons learned from the microarray community. Am J Surg Pathol 2011; 35: 783-786.

54. Hipp JD, Sica J, McKenna B et al. The need for the pathology community to sponsor a whole slide imaging repository with technical guidance from the pathology informatics community. J Pathol Inform 2011;2:31.

55. Mroz P, Parwani AV, Kulesza P. Central pathology review for phase III clinical trials: the enabling effect of virtual microscopy. Arch Pathol Lab Med 2013; 137: 492-495. 